\title{
Survival resonances in an atom-optics system driven by temporally and spatially periodic dissipation
}

\author{
Shijie Chai, Julia Fekete, Peter McDowall, Simon Coop, Thue Lindballe, and Mikkel F. Andersen* \\ The Dodd-Walls Centre for Photonics and Quantum Technologies, Department of Physics, University of Otago, 9054 Dunedin, New Zealand
}

(Received 13 November 2017; published 22 March 2018)

\begin{abstract}
We investigate laser-cooled atoms periodically driven by pulsed standing waves of light tuned close to an open atomic transition. This nonunitary system displays survival resonances for certain driving frequencies. The survival resonances emerge as a result of the matter-wave Talbot-Lau effect, similar to the Talbot effect causing quantum resonances in the atom optics $\delta$-kicked rotor. Since the Talbot-Lau effect occurs for incoherent waves, the survival resonances can be observed using thermal atoms. A microlensing effect can enhance the height and incisiveness of the resonances. This may find applications in precision measurements.
\end{abstract}

DOI: 10.1103/PhysRevA.97.033616

\section{INTRODUCTION}

Periodically driven one-dimensional systems play a central role in studies of nonlinear dynamics. They exhibit a wide range of dynamical phenomena and are the simplest systems to display chaotic behavior. An important experimental platform among such systems is the atom-optics $\delta$-kicked rotor (AODKR) [1]. It consists of a quantum particle that receives $\delta$ kicks at regular time intervals from a spatially periodic potential. Its rich dynamics has enabled clear experimental investigations of a prodigious range of quantum effects. Specifically, experiments have shown that particular combinations of parameters and initial conditions can lead to directional transport in symmetric systems [2,3] and hierarchies of quantum resonances (QRs) and quantum accelerator modes, where the transfer of energy to the particle is unbounded [4-9]. The AODKR's sub-Fourier characteristics and ability to generate coherent superpositions of states with highly different momenta have led to suggestions that it can be applied as a tool in atom interferometry and precision measurements, such as the determination of the fine structure constant $\alpha$ or the local gravitational field [9-17].

The $\delta$ kicks in the AODKR cause a spatially periodic phase modulation of the particle's wave function, which leads to splitting of an initial momentum eigenstate into multiple diffraction orders. In this paper, we introduce an extension to the standard AODKR. In addition to a phase modulation, the kicks also modulate the wave function's amplitude by adding spatially periodic loss. In terms of the classical rotor [18], our alternative approach gives each kick a finite probability of "survival" or "death" of the rotor that depends on its angle at the time of the kick. The system could therefore be deemed as the " $\delta$-killed rotor." The system is nonunitary and a meaningful dynamic observable is the survival probability of the rotor or particle. We show that the survival probability exhibits a series of resonances for certain kicking periods, that is, for particular time intervals between the kicks. The survival resonances relate to the QRs known from the standard AODKR. However,

\footnotetext{
*mikkel.andersen@otago.ac.nz
}

the physical principles underpinning the resonance effects are distinct. The appearance of standard QRs is based on phase pattern revivals due to the matter-wave Talbot effect [19] and is highly sensitive to the initial momentum of the atoms $[6,20]$. This requires heavy initial momentum selection; otherwise, the resonant atoms are observed on a large background of nonresonant atoms, when using an initial source of thermal atoms $[8,9,17]$. In contrast, survival resonances are based on the matter-wave Talbot-Lau effect [21], which allows for their observation using incoherent atoms without a large unwanted background.

We study the survival resonances for different experimental parameters and how their shape evolves as a function of time. The height of the resonances is enhanced by a microlensing effect [22] originating from the still present phase modulation of the wave function. The microlensing effect also affects the shape of the survival resonances, resulting in very sharp features that are ideal for precision measurements. Furthermore, the height of the resonances decays in the long time limit, showing that the resonant phenomenon is a transient effect. The experimental results generally agree well with a model based on the one period time evolution operator that is characteristic of the periodically driven system.

The structure of the paper is the following. Section II describes the experimental system and how to model it. Section III shows measurements and calculations of the atomic survival probability as a function of different parameters, while Sec. IV provides a general discussion and conclusions.

\section{THE SYSTEM}

We experimentally realize the $\delta$-killed rotor by exposing a cloud of laser-cooled atoms to a sequence of optical standingwave pulses. We ignore interactions between the atoms, which is valid for a dilute gas. As shown in Fig. 1(a), we treat the atom (of mass $M$ ) as a three-level system, consisting of an initial hyperfine ground state $|g\rangle$, a dark hyperfine ground state $\left|g_{D}\right\rangle$, and an excited state $|e\rangle$. We consider the atom to be lost if it goes to the dark state $\left|g_{D}\right\rangle$. The optical standing wave is formed by retroreflecting a laser beam that has an angular frequency $\omega_{L}$ and wave number $k_{L} \cdot \omega_{L}$ is tuned within two natural line widths 


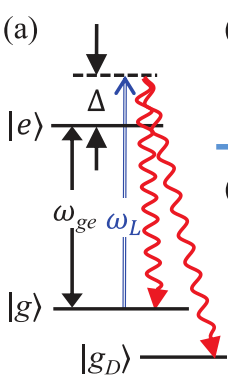

(b)

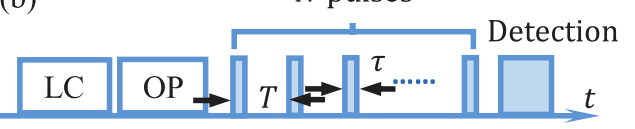

(c)

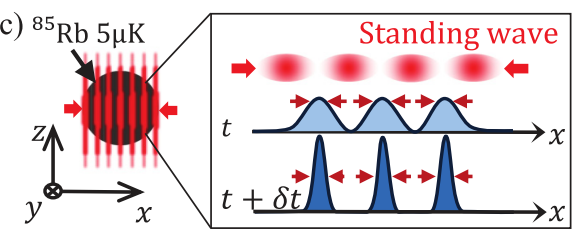

FIG. 1. (a) Simplified energy level diagram. The blue arrow represents the absorption of a photon and the wavy lines represent the spontaneous emission. (b) Time sequence of the experiment (not to the scale), with LC for laser cooling and OP for optical pumping stages. (c) Microlensing effect due to the optical dipole force. A blue detuned standing wave acts as an array of lenses causing the atomic density distribution (lower panel) to form a narrower peak at time $\delta t$ after the application of the pulse.

$\Gamma$ of the open transition from the ground state $|g\rangle$ to the excited state $|e\rangle$. We use short standing-wave pulses, which allow us to neglect the evolution caused by the kinetic energy term for these brief durations (Raman-Nath approximation). Thus, by using dipole and rotating-wave approximations, the dynamics during the pulse is described by the Hamiltonian [23]:

$$
\begin{aligned}
H= & -\left(\hbar \Delta+\frac{i \hbar \Gamma}{2}\right)|e\rangle\langle e| \\
& +\frac{\hbar \Omega_{0}}{2} \cos \left(k_{L} x\right)(|e\rangle\langle g|+| g\rangle\langle e|),
\end{aligned}
$$

where $x$ is the atomic position operator and $\Delta \equiv \omega_{L}-\omega_{g e}$ is the laser detuning $\left(\omega_{g e}\right.$ denotes the atomic transition frequency). $\Omega_{0}$ is the on resonance Rabi frequency at the antinodes of the standing wave for the $|g\rangle$ to $|e\rangle$ transition. The non-Hermitian decay term $-(i \hbar \Gamma / 2)$ accounts for the relaxation from the excited state into $\left|g_{D}\right\rangle$. We thereby neglect the spontaneous decay into the state $|g\rangle$ that causes an additional incoherent background.

The light field of the standing wave effectively acts as a diffraction grating which modulates both the phase and amplitude of the atomic wave function. We can define a "grating operator" $G$, which describes the effect of a standing-wave pulse on the wave function of atoms remaining in $|g\rangle$. Equation (1) gives $G=(1-$ $\left.\lambda_{2} / \lambda_{1}\right)^{-1}\left[\exp \left(-i \lambda_{2} \tau / \hbar\right)-\lambda_{2} \exp \left(-i \lambda_{1} \tau / \hbar\right) / \lambda_{1}\right] . \quad \lambda_{1,2}=$ $-(\hbar \Delta+i \hbar \Gamma / 2) / 2 \mp \sqrt{(\hbar \Delta+i \hbar \Gamma / 2)^{2}+\hbar^{2} \Omega_{0}^{2} \cos ^{2}\left(k_{L} x\right)} / 2$ are the eigenvalues of $H$ and $\tau$ is the pulse duration.

In the case of a far-off-resonance standing wave $(\Delta \gg \Gamma)$, the light field yields a conservative spatially periodic potential. $G$ reduces to the familiar unitary phase grating operator $G=\exp \left[-i \phi_{d} \cos \left(2 k_{L} x\right)\right]\left(\phi_{d}=\Omega_{0}^{2} \tau / 8 \Delta\right)$ from the standard AODKR [9]. When the standing wave is resonant $(\Delta=0)$, $G$ is an absorption grating, which depletes the population of the initial ground state everywhere except in the vicinity of the nodes; i.e., it modulates the amplitude of the atomic wave function [24]. The population in the initial ground state is therefore not conserved and the system is dissipative. For standing-wave light with a frequency close to an open atomic transition, $G$ simultaneously modulates the phase and amplitude of the atomic wave function. Thus, we can write $G=A(x) \exp [i \phi(x)]$, where $A(x) \in[0,1]$ and $\phi(x) \in[0,2 \pi)$ for all $x$.

The temporal periodicity of the system allows us to construct a one-period time-evolution operator $F$ that governs the dynamics of the wave function of the atoms that remain in $|g\rangle$ :

$$
F=\exp \left[-\frac{i}{\hbar} \frac{p^{2}}{2 M}(T-\tau)\right] G,
$$

where $p$ is the atomic momentum operator and $T$ is the kicking period. The exponential operator arises from the free evolution between pulses, and $G$ gives the time evolution caused by the standing-wave pulse. The atomic wave function after $N$ standing-wave pulses is then simply given by $\left|\psi_{N}\right\rangle=F^{N}\left|\psi_{0}\right\rangle$, where $\left|\psi_{0}\right\rangle$ is the initial wave function. Since $G$ is nonunitary, so is this evolution.

In our experiments, we use ${ }^{85} \mathrm{Rb}$ atoms and their $F=3$ and $F=2$ hyperfine ground states as $|g\rangle$ and $\left|g_{D}\right\rangle .|e\rangle$ is the $F^{\prime}=3$ excited state on the D2 transition. As illustrated in Fig. 1(b), we first laser cool a cloud of atoms to a temperature of $5 \mu \mathrm{K}$ and optically pump them into the $F=3$ ground state $|g\rangle$. We then expose them to a sequence of $N$ brief optical standing-wave pulses with kicking period $T$. The pulse duration $\tau$ is $300 \mathrm{ns,}$ which fulfills the Raman-Nath condition. The standing-wave light propagates horizontally along the $x$ axis [see Fig. 1(c)]. It is formed by retroreflecting a linearly polarized (along $z$ ) laser beam of diameter $3.5 \mathrm{~mm}\left(1 / e^{2}\right)$ and power of $3 \mathrm{~mW}$. When the standing-wave light excites an atom to the $F^{\prime}=3$ state, it can spontaneously decay into the $F=2$ ground state $\left|g_{D}\right\rangle$. During the pulse sequence, a bias magnetic field of $5.6 \mathrm{G}$, along the $y$ axis, quenches the dark state in the $F=3$ ground-state manifold. We measure the survival probability (the proportion of atoms surviving in the $F=3$ ground state) immediately after the pulse sequence by detecting fluorescence while exciting them on the closed $F=3$ to $F^{\prime}=4$ transition on the D2 line.

\section{RESULTS}

To explore the system, we measured the survival probability while varying experimental parameters.

\section{A. Survival resonances}

Figure 2(a) displays the result of varying the kicking period between the pulses for $N=7$ and $\Delta / 2 \pi=-10 \mathrm{MHz}$. It shows a series of survival resonances for specific values of the time interval. These are separated by half the Talbot time $T_{\mathrm{T}} / 2=32.4 \mu \mathrm{s}$ (where $T_{\mathrm{T}}=\pi M / \hbar k_{L}^{2}=64.8 \mu \mathrm{s}$ is the Talbot time for ${ }^{85} \mathrm{Rb}$ atom [20]). The emergence of the survival resonances can be conceptually understood through the matter-wave Talbot-Lau effect $[21,25]$, which is illustrated in Fig. 2(b). When an incoherent atomic wave impinges on two identical absorption gratings separated by a time $T$, a density modulation of the atomic cloud can be observed at time $T$ after the second grating [21]. Figure 2(b) shows the atomic density distribution at time $T$ after the second grating as $T$ is changed. When the time between the gratings is an integer multiple of half the Talbot time $n T_{\mathrm{T}} / 2$, where $n \in \mathbb{N}$, then a 
(a)

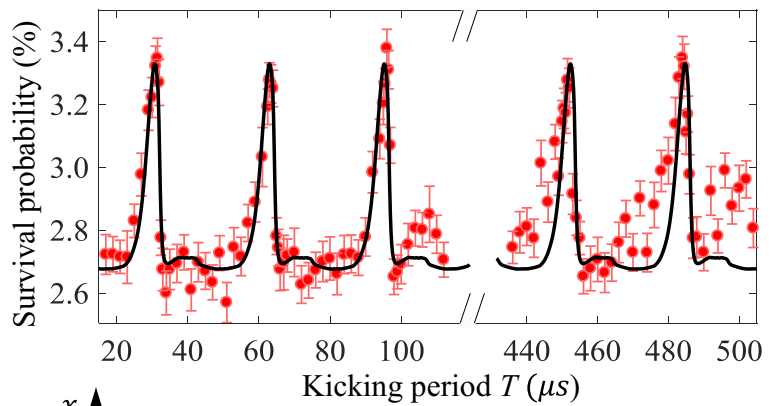

(b)

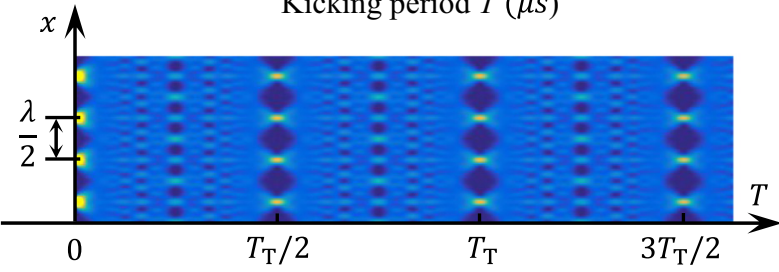

FIG. 2. (a) The survival probability as a function of kicking period $T$ after a train of $N=7$ pulses. Each point is the mean of 90 experimental runs and the error bars show the standard deviation of the mean. The solid line is the calculation based on Eq. (2). (b) Matter-wave Talbot-Lau interference. The color map represents the calculated atomic density distribution at time $T$ after two pulses separated by $T$. The horizontal axis is $T$, and the vertical axis is the coordinate along the standing wave with $\lambda$ being the wavelength of the light.

grating-like spatial atomic distribution will occur at this time. This density pattern is a near perfect "self-image" of the atomic distribution after the second grating. If a third standing-wave pulse is applied when this high-contrast distribution forms, then the atoms have a high probability of surviving it since most of them are localized around the nodes. This effect leads to the survival resonances in Fig. 2(a). From the third pulse and onward, the previous two pulses will ensure that the atoms are near the nodes of the standing wave when $T=n T_{\mathrm{T}} / 2$. Figure 2(a) displays five such survival resonances, the first three of which are for $n=1$ to 3 , while the last two are for $n=14$ and 15 , showing that the phenomena persist for large pulse separations.

In the standard $\delta$-kicked rotor, QRs occur as a result of the matter-wave Talbot effect, which for resonant condition leads to revivals of the spacial phase patterns imprinted by previous kicks at the time of the next kick. QRs thereby manifest themselves as unbounded transfer of kinetic energy due to the resulting linear increase in the amplitude of the spacial phase modulation with pulse number $N[6,19]$. The survival resonances are therefore intimately related to QRs in the standard $\delta$-kicked rotor. However, instead of the spacial phase pattern revivals the Talbot-Lau revivals of the density pattern leads to enhanced survival.

The solid line in Fig. 2(a) represents a numerical calculation where an ensemble of initially Gaussian wave packets are evolved using Eq. (2). The widths of the Gaussian wave packets correspond to the initial temperature, and the ensemble covers a period of the standing wave homogeneously. We account for an imbalance of the standing-wave beam intensities using measured losses from the viewports and the retromirror and fit the initial standing-wave beam intensity. An offset has been

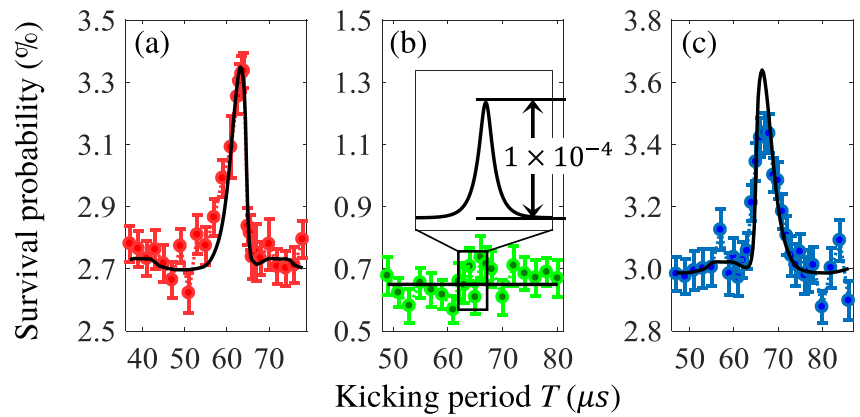

FIG. 3. Survival resonance for different standing-wave detunings. Measured data (markers with error bars) and calculations (solid lines) for survival resonance at the first Talbot time. (a) Red detuning: $\Delta / 2 \pi=-10 \mathrm{MHz}$, (b) resonant light: $\Delta=0 \mathrm{MHz}$ (the inset shows the calculation on a magnified scale), and (c) blue detuning: $\Delta / 2 \pi=$ $10 \mathrm{MHz}$.

added to the calculation to account for spontaneous decay back to the $F=3$ ground state. The calculation agrees well with the data and the fitted intensity agrees with the one estimated from the beam power and size within $25 \%$. This confirms that the model captures the dynamics of the system for the parameters used. Calculations in the following figures are done in a similar manner, but using the beam intensity found in Fig. 2(a). For off-resonance kicking periods longer than $100 \mu \mathrm{s}$, the experimental data deviate from the numerical calculation. Experimental effects that are not included in the model which could contribute to this include low-frequency phase noise of the standing wave due to vibrations of the retroreflecting mirror, the atoms' transverse motion in the standing wave beam which could contain phase distortions, and small residual forces on the atoms, for example, originating from stray magnetic field gradients.

The comb of survival resonances in Fig. 2(a) bears similarities to the equally spaced frequency resonances in a FabryPerot interferometer. In both cases, the resonances occur due to multipath interference. Each standing-wave pulse diffracts a wave function into a number of diffraction orders. The survival resonances emerge when diffraction orders originating from the same initial state constructively interfere at the nodes of subsequent standing-wave pulses. The system is therefore a many-path atom interferometer.

\section{B. Effect of microlensing}

In addition to modulating the amplitude of the atomic wave function, the standing-wave pulses also modulate its phase. The relative strengths of these effects can be changed by changing the detuning $\Delta$ of the standing wave. Figure 3 shows the survival resonance at the Talbot time $T_{\mathrm{T}}(n=2)$ for three different detunings $(\Delta / 2 \pi=0$ and $\pm 10 \mathrm{MHz})$ with $N=7$. When the standing-wave light is detuned [Figs. 3(a) and 3(c)], the line shape of the survival resonances is asymmetric with a steep edge on one side and a smooth edge on the other. Meanwhile, the peak survival is obtained for $T$ slightly below or above the exact Talbot time $T_{\mathrm{T}}$ for red or blue detuned light, respectively. These effects originate from the phase modulation of the atomic wave function, which gives rise to a microlensing 
effect. When the optical frequency of the standing wave is tuned above the atomic transition, the optical dipole force directs atoms towards the low-intensity regions (the standingwave nodes). The atoms remaining in the ground state $|g\rangle$ will therefore form tight foci at a time $\delta t$ after each pulse [see Fig. 1(c)], which offsets the survival resonance slightly from $T_{\mathrm{T}}$ as seen in Fig. 3(c). We note that a similar microlensing effect has previously been used to realize nanolithography by producing a periodic array of localized metastable atoms [22]. When the standing wave is red detuned [Fig. 3(a)], the optical dipole force points away from the standing-wave nodes, causing the survival resonance peak to shift oppositely. For a resonant standing wave $(\Delta=0)$, the survival resonance is symmetric and centered at $T=T_{\mathrm{T}}$ as shown in the inset of Fig. 3(b), but for the measured data in Fig. 3(b), the peak height is below the noise level.

Comparing the measured data in Fig. 3 to the calculation (solid lines), we see a good agreement for red detuning while there is a discrepancy in the peak heights for the bluedetuned standing wave. This could be due to the two-level approximation of Eq. (1), since the presence of additional excited hyperfine states makes spontaneous emission and phase modulation of the atomic wave function differ in magnitude between red and blue detunings.

A useful feature in Fig. 3 is that the survival resonance provides a steep edge when the standing wave is not at resonance. This allows for precise determination of the period of a signal like that in Fig. 2(a), and thereby of the recoil frequency $\omega_{r}=\pi / 2 T_{\mathrm{T}}$ [26] (which in turn can be used to determine the fine structure constant $\alpha$ [27]). The increase in precision yielded by the steep edges results from the enhancement of high-order diffraction due to the phase modulation provided by the off-resonance standing wave. In this way, using nearresonant light provides a way of getting a high sensitivity (steep edge) without losing signal size (peak height).

\section{Pulse number evolution}

To further understand the dynamics of the system, we now study how the survival resonances evolve with the pulse number $N$. We determine the trends of the peak height and width on the steep side of the survival resonance by fitting a two-piece normal distribution to both the calculated and measured data. Figure 4 shows the resulting steep-side standard deviations (a) and peak heights (b) for the peak at the Talbot time $(n=2)$ and $\Delta / 2 \pi=-10 \mathrm{MHz}$.

In Fig. 4(a), we see that the widths of the resonances monotonically decrease with increasing $N$. This is not surprising since a higher $N$ leads to population of higher diffraction orders that accumulate phases at higher rates during the free evolutions. This results in a more stringent requirement on the free evolution time to guarantee that the different diffraction orders are in phase to produce sharp coherence echoes at the times of the pulses. For comparison, the Fourier limit states that the time required to distinguish two close frequencies is inversely proportional to the frequency difference $\Delta \omega$ [16]. This gives that the time required to discriminate the periods of two signals is also inversely proportional to their difference. Figure 4(a) shows that the calculated data is well fitted by a power law (blue line), but the power is not $N^{-1}$ (shown

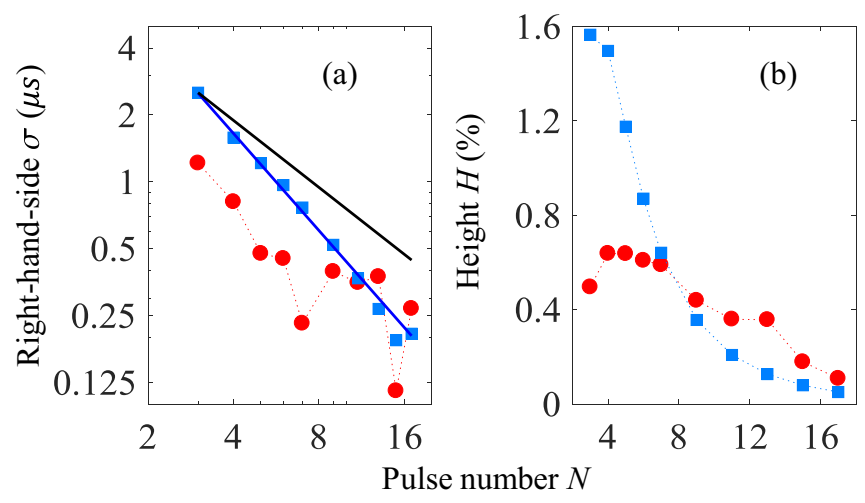

FIG. 4. Measured (red circles) and calculated (blue squares) steep-side standard deviation (plotted using double logarithmic scales) (a) and height (b) of the survival resonance as a function of $N$. The blue line in panel (a) is a power-law fit to the calculation while the black line is a curve proportional to $1 / N$ for comparison. The standing wave is red detuned $10 \mathrm{MHz}$ from the $F=3$ to $F^{\prime}=3$ atomic transition, similar to Fig. 2(a).

as black line) as expected from the Fourier relation. Recall that the experiment duration is approximately proportional to $N$ in the large- $N$ limit. The fitted power of -1.45 therefore shows that the system exhibits sub-Fourier behavior [28] as is also seen with the standard AODKR $[11,16]$. The experimental data initially displays a similar power relation, until the width gets so small that the signal-to-noise ratio is poor.

In Fig. 4(b), we see a significant discrepancy between calculated and measured peak heights for $N<6$. In this region, there is a significant offset present (up to 16\%) in the measured data due to spontaneous decay back to the $F=3$ ground state, which may contribute to the discrepancy. Additionally, for small $N$ the survival probability as a function of $T$ has a more complex behavior than clearly separated resonances, but we do not expect this to contribute to the discrepancy since the height evaluation method is the same for the experimental data and the calculation. For $N>6$, the agreement between calculation and measurement is better. A decaying peak height (for large $N$ ) is expected since each standing-wave pulse will continue to remove atoms from the $F=3$ ground state due to the imbalance of the standing-wave intensities, resulting in the nodes having finite intensity. For a perfectly balanced standing wave, calculations show that we still see a decay, albeit at a significantly lower rate. This is because the atomic distributions at the time of pulses have a finite width.

In order to clearly observe resonance peaks for a given detuning and pulse number, the standing-wave intensity should be chosen in a range that occurs as a trade-off between two effects. If the intensity is too low, one simply sees a high survival for all driving periods. On the other hand, if a too high intensity is used, the height of the peaks become too small to observe. Higher peaks for large $N$ in Fig. 4(b) can therefore be observed simply by reducing the standing-wave intensity.

\section{DISCUSSION AND CONCLUSIONS}

An intriguing feature of the system is its relative technical simplicity. The light for the standing wave is derived 
from the same laser that is used for laser cooling the initial atomic sample, and the experiment output is a simple internal state detection. In future work, it would therefore be interesting to investigate how to use the survival resonances for gravity measurements, since the simplicity of our system makes it a good candidate for a compact atomic gravimeter.

To conclude, we have studied an alternative approach to the quantum $\delta$-kicked particle in which the amplitude of the wave function is modulated in addition to the usual phase modulation. The survival probability of the particle during each kick is therefore dependent on its position in the standing wave. This dissipative system displays a comb of narrow survival resonances separated by $T_{\mathrm{T}} / 2$ when the kicking period is scanned. The survival resonances are a consequence of the matter-wave Talbot-Lau effect and can therefore easily be observed with an incoherent ensemble of particles. The phase modulation causes a microlensing effect that focuses the particles to positions with high survival probability, and thereby enhances the height of the survival resonances. The relatively simple experimental construction combined with the sharp features may make the resonances a useful tool in future precision measurements and experiments.

\section{ACKNOWLEDGMENT}

This work was supported by the NZ-MBIE (Contract No. UOOX1402).
[1] F. L. Moore, J. C. Robinson, C. Bharucha, P. E. Williams, and M. G. Raizen, Phys. Rev. Lett. 73, 2974 (1994).

[2] M. Sadgrove, M. Horikoshi, T. Sekimura, and K. Nakagawa, Phys. Rev. Lett. 99, 043002 (2007).

[3] I. Dana, V. Ramareddy, I. Talukdar, and G. S. Summy, Phys. Rev. Lett. 100, 024103 (2008).

[4] M. K. Oberthaler, R. M. Godun, M. B. d'Arcy, G. S. Summy, and K. Burnett, Phys. Rev. Lett. 83, 4447 (1999).

[5] J. F. Kanem, S. Maneshi, M. Partlow, M. Spanner, and A. M. Steinberg, Phys. Rev. Lett. 98, 083004 (2007).

[6] C. Ryu, M. F. Andersen, A. Vaziri, M. B. d'Arcy, J. M. Grossman, K. Helmerson, and W. D. Phillips, Phys. Rev. Lett. 96, 160403 (2006).

[7] G. Behinaein, V. Ramareddy, P. Ahmadi, and G. S. Summy, Phys. Rev. Lett. 97, 244101 (2006).

[8] M. B. d'Arcy, R. M. Godun, M. K. Oberthaler, D. Cassettari, and G. S. Summy, Phys. Rev. Lett. 87, 074102 (2001).

[9] W. H. Oskay, D. A. Steck, V. Milner, B. G. Klappauf, and M. G. Raizen, Opt. Commun. 179, 137 (2000).

[10] M. Sadgrove and S. Wimberger, Adv. At. Mol. Opt. Phys. 60, 315 (2011).

[11] I. Talukdar, R. Shrestha, and G. S. Summy, Phys. Rev. Lett. 105, 054103 (2010).

[12] B. Daszuta and M. F. Andersen, Phys. Rev. A 86, 043604 (2012).

[13] P. McDowall, A. Hillard, M. McGovern, T. Grünzweig, and M. F. Andersen, New J. Phys. 11, 123021 (2009).

[14] R. A. Horne, R. H. Leonard, and C. A. Sackett, Phys. Rev. A 83, 063613 (2011).
[15] J. Ni, W. K. Lam, S. Dadras, M. F. Borunda, S. Wimberger, and G. S. Summy, Phys. Rev. A 94, 043620 (2016).

[16] P. Szriftgiser, J. Ringot, D. Delande, and J. C. Garreau, Phys. Rev. Lett. 89, 224101 (2002).

[17] J. Fekete, S. Chai, S. A. Gardiner, and M. F. Andersen, Phys. Rev. A 95, 033601 (2017).

[18] E. Ott, Chaos in Dynamical Systems (Cambridge University Press, Cambridge, UK, 2002).

[19] M. Lepers, V.Zehnlé, and J. C. Garreau, Phys. Rev. A 77, 043628 (2008).

[20] L. Deng, E. W. Hagley, J. Denschlag, J. E. Simsarian, M. Edwards, C. W. Clark, K. Helmerson, S. L. Rolston, and W. D. Phillips, Phys. Rev. Lett. 83, 5407 (1999).

[21] A. Turlapov, A. Tonyushkin, and T. Sleator, Phys. Rev. A 71, 043612 (2005).

[22] K. S. Johnson, J. H. Thywissen, N. H. Dekker, K. K. Berggren, A. P. Chu, R. Younkin, and M. Prentiss, Science 280, 1583 (1998).

[23] S. Fray, C. A. Diez, T. W. Hänsch, and M. Weitz, Phys. Rev. Lett. 93, 240404 (2004).

[24] A. Turlapov, A. Tonyushkin, and T. Sleator, Phys. Rev. A 68 023408 (2003).

[25] A. D. Cronin, J. Schmiedmayer, and D. E. Pritchard, Rev. Mod. Phys. 81, 1051 (2009).

[26] S. Beattie, B. Barrett, I. Chan, C. Mok, I. Yavin, and A. Kumarakrishnan, Phys. Rev. A 79, 021605 (2009).

[27] R. Bouchendira, P. Cladé, S. Guellati-Khélifa, F. Nez, and F. Biraben, Phys. Rev. Lett. 106, 080801 (2011).

[28] F. S. Cataliotti, R. Scheunemann, T. W. Hänsch, and M. Weitz, Phys. Rev. Lett. 87, 113601 (2001). 